\title{
On Darcy-Brinkman Equation: Viscous Flow Between Two Parallel Plates Packed with Regular Square Arrays of Cylinders
}

\author{
Haidong Liu, Prabhamani R. Patil ${ }^{\#}$ and Uichiro Narusawa * \\ Department of Mechanical \& Industrial Engineering, Northeastern University, Boston, Massachusetts \\ 02115, U.S.A. \\ \# Permanent address: Anna University, Chennai, India \\ * Author to whom correspondence should be addressed. E-Mail: narusawa@coe.neu.edu
}

Received: 12 December 2006 / Accepted: 5 September 2007 / Published: 11 September 2007

\begin{abstract}
Effects of the bounding solid walls are examined numerically for slow flow over regular, square arrays of circular cylinders between two parallel plates. A local magnitude of the rate of entropy generation is used effectively to determine the flow region affected by the presence of the solid boundary. Computed axial pressure gradients are compared to the corresponding solution based on the Darcy-Brinkman equation for porous media in which the effective viscosity appears as an additional property to be determined from the flow characteristics. Results indicate that, between two limits of the Darcian porous medium and the viscous flow, the magnitude of $\hat{\mu}$ (the ratio of the effective viscosity to the fluid viscosity) needs to be close to unity in order to satisfy the non-slip boundary conditions at the bounding walls. Although the study deals with a specific geometric pattern of the porous structure, it suggests a restriction on the validity of the Darcy-Brinkman equation to model high porosity porous media. The non-slip condition at the bounding solid walls may be accounted for by introducing a thin porous layer with $\hat{\mu}=1$ near the solid walls.
\end{abstract}

Keywords: entropy generation, porous media, Darcy-Brinkman equation, effective viscosity. 


\section{Nomenclature}

$\begin{array}{ll}C & \text { solid fraction }(=1-\phi) \\ D a & H / \sqrt{K} \\ f(C) & \text { function in Eq.(7) } \\ \mathrm{H} & \text { channel half-depth } \\ K & \left.\text { permeability [ } \mathrm{m}^{2}\right] \\ l & \text { half of the side of a unit cell ( Figure 1(b)) } \\ L & \text { depth of the top and the bottom layer in three layer model (Figure 7) } \\ p & \text { pressure } \\ q & \text { volume flow rate per channel depth } \\ \dot{S}_{G, c e l l}^{\prime} & \text { rate of entropy generation over a unit cell per channel depth } \\ \dot{S}_{G}^{\prime \prime \prime} & \text { rate of entropy generation per channel width } \\ T & \text { temperature [K] } \\ u & \text { x-direction velocity of viscous flow } \\ \bar{u} & u_{s} / u_{m} \\ u_{m} & \text { mean velocity (=q/2H) } \\ u_{s} & \text { superficial velocity in porous media } \\ v & \text { y-direction velocity of viscous flow } \\ \vec{V} & \text { velocity vector } \\ x & \text { axial (flow direction, longitudinal coordinate) } \\ y & \text { lateral coordinate } \\ \tilde{y} & y / H\end{array}$

Greek Symbols

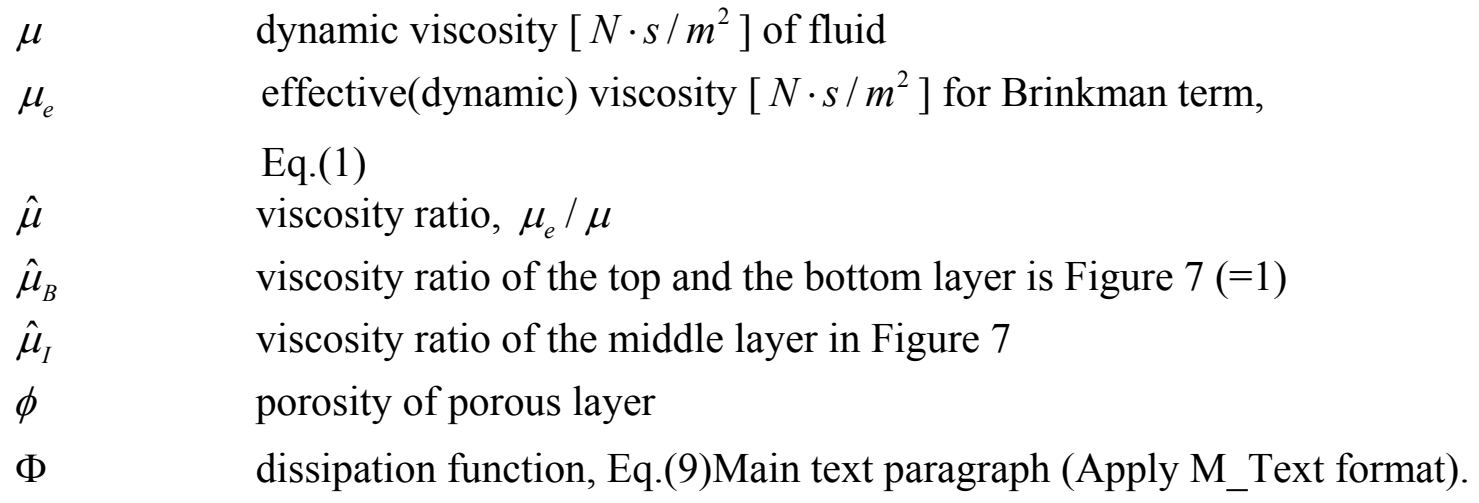

\section{Introduction}

The Darcy-Brinkman equation is a governing equation for flow through a porous medium with an extra Laplacian (viscous) term (Brinkman term) added to the classical Darcy equation. The equation has been used widely to analyze high-porosity porous media. The dynamic viscosity, $\mu_{e}$, associated with the Brinkman term is referred to as the effective viscosity. Studies in the past yielded varying results for the magnitude of the viscosity ratio, $\hat{\mu}$ ( $=\mu_{e} / \mu$ with $\mu=$ fluid viscosity) between slightly 
less than unity to as high as $<\sim 10$ for high porosity porous media [3,5,8-10]. The validity of the Darcy- Brinkman equation has also been a subject of investigation, particularly in relation to the boundary conditions at the solid- as well as fluid- interface $[11,12]$. In the present study our subject of interest is flow over regular square arrays of circular cylinders bounded at the top and the bottom by solid plates. The flow in the absence of the bounding walls is studied in detail by Sangani and Acrivos [15]. The analysis which solves the Navier- Stokes equations rather than the Darcy equation, yields a relation between the permeability of the regular array structure and the porosity (volume fraction occupied by the flow), confirming that the Darcy equation is valid for flow through regular structures over the whole spectrum of the porosity. Therefore, quantitative relations between the wall effects and the Darcy-Brinkman equation may be examined in a more focused manner through the present investigation. One of the objectives of the present analysis is to examine flow structure near the bounding walls. It is generally accepted that the effects of a solid boundary is confined within a thin boundary layer [12]. Our second objective is to test the feasibility of addressing the thin region by using the Darcy-Brinkman equation. The Darcy-Brinkman equation in recent years is employed in biomedical hydrodynamic studies [7], including its use in modeling a thin fibrous surface layer coating blood vessels (endothelial surface layer) as it is a highly permeable, high porosity porous medium $[13,14,17]$. A better understanding of the characteristics of the Darcy-Brinkman equation, therefore, is an important part of more practical problems; thus forming a motivation of the present report.

\section{Analyses}

As shown in Figure 1, we consider a steady, incompressible, fully-developed, very slow ( Re (Reynolds number) $\rightarrow 0$ ) flow across regular square arrays of circular cylinders, bounded by parallel plates. Governing equations based on the Darcy-Brinkman equation for porous media and the boundary conditions are,

$$
\frac{d p}{d x}=-\frac{\mu}{K} u_{s}+\mu_{e} \frac{d^{2} u_{s}}{d y^{2}}, \quad u_{s}(y=H)=0, \quad \frac{d u_{s}}{d y}(y=0)=0
$$

where $p=$ pressure, $u_{s}=$ superficial velocity, $K=$ permeability of porous medium. In terms of non-dimensional variables defined as, $\tilde{y}=y / H, \tilde{u}=u_{s} / u_{m}\left(u_{m}=\right.$ mean velocity $=q / 2 H, q=$ volume flow rate per channel width), the formulation and its solution become,

$$
\begin{aligned}
\frac{H^{2}}{\mu_{e} u_{m}} \cdot\left(-\frac{d p}{d x}\right) & =\frac{1}{\hat{\mu}} \frac{H^{2}}{K} \tilde{u}-\frac{d^{2} \tilde{u}}{d \tilde{y}^{2}}, \quad \tilde{u}(\tilde{y}=1)=0, \quad \frac{d \tilde{u}}{d \tilde{y}}(\tilde{y}=0)=0 . \\
\tilde{u} & =\frac{K}{\mu u_{m}}\left(-\frac{d p}{d x}\right)\left[1-\frac{\cosh (D a \cdot \tilde{y} / \sqrt{\hat{\mu}})}{\cosh (D a / \hat{\mu})}\right]
\end{aligned}
$$

where $\hat{\mu}=\mu_{e} / \mu$ (viscosity ratio), $D a=H / \sqrt{K}$. 


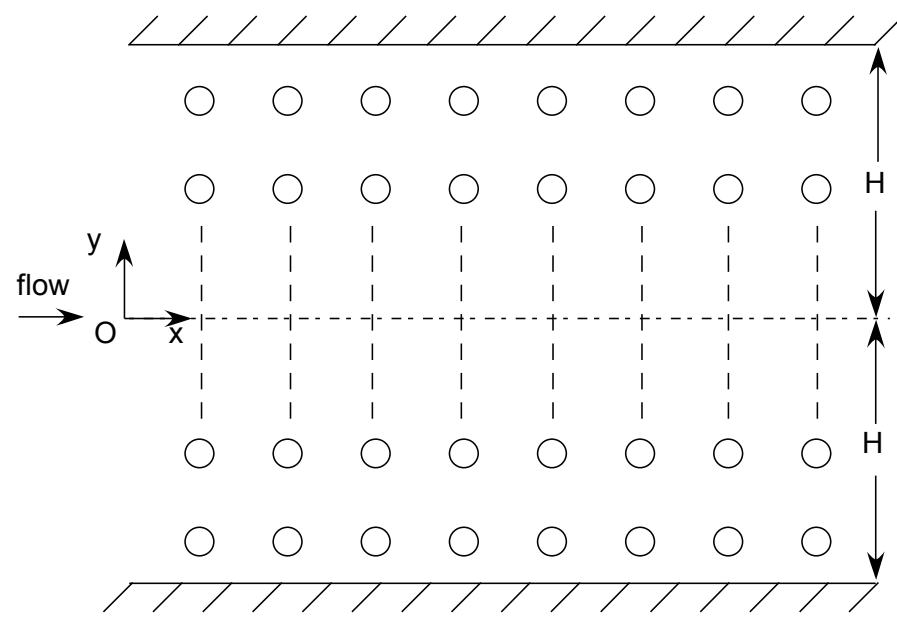

(a)

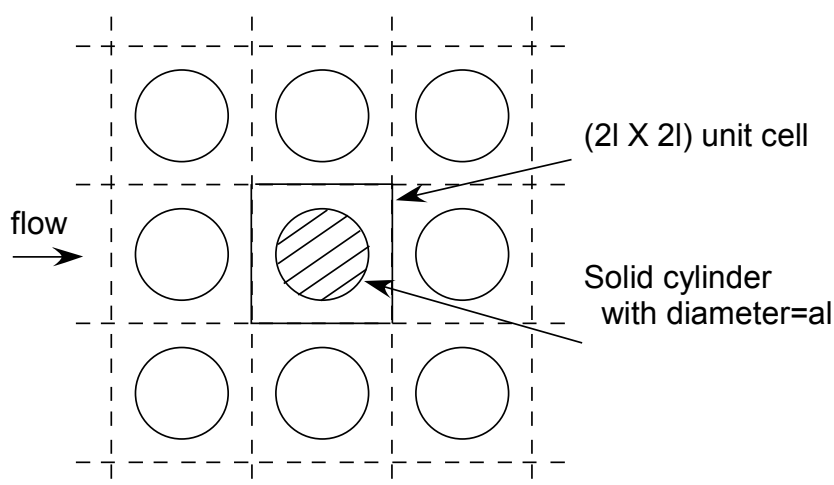

(b)

Figure 1. (a) Flow between two parallel plates filled with regular square arrays of circular cylinders,

(b) Regular square arrays of circular cylinders.

Upon an integration of the governing equation, Eq.(2a), over the channel half-depth, $H$,

$$
\operatorname{noting} \int_{0}^{1} \tilde{u} d \tilde{y}=1\left(u_{m}=\int_{0}^{H} u d y / H\right)
$$

we obtain the following equation for the pressure gradient;

$$
\frac{H^{2}}{\mu u_{m}} \cdot\left(-\frac{d p}{d x}\right)=\frac{D a^{2}}{1-\frac{\tanh (D a / \sqrt{\hat{\mu}})}{D a / \sqrt{\hat{u}}}}
$$

Since $\lim _{x \rightarrow 0} \tanh x \approx x-x^{3} / 3$, the viscous flow limit is reached as $K \rightarrow \infty,(D a \rightarrow 0)$,

$$
\frac{H^{2}}{\mu u_{m}} \cdot\left(-\frac{d p}{d x}\right)_{\text {viscous limit }}=3 \hat{\mu} \text {. }
$$

The viscous flow limit corresponds to the case of $\hat{\mu}=1$ in the equation above.

The Darcian limit of flow through a porous medium is recovered as $K \rightarrow 0,(D a \rightarrow \infty)$

$$
\frac{H^{2}}{\mu u_{m}} \cdot\left(-\frac{d p}{d x}\right)_{\text {Darcian Limit }}=\frac{H^{2}}{K}=D a^{2} .
$$

For the case of our interest, the porous medium consisting of regular square arrays of cylinders, the permeability, $K$, may be expressed as,

$$
K=\frac{(2 l)^{2}}{f(C)}, \quad \text { that is, } D a=\sqrt{f(C)} \cdot\left(\frac{H}{2 l}\right)
$$


where $C$ (solid fraction) $=1-\phi(\phi=$ porosity), $2 l=$ side length of a single square unit. (See Figure 1.) The function, $f(C)$, is given in an explicit form for high porosity arrays and in a graphical form for low porosity arrays in [15].

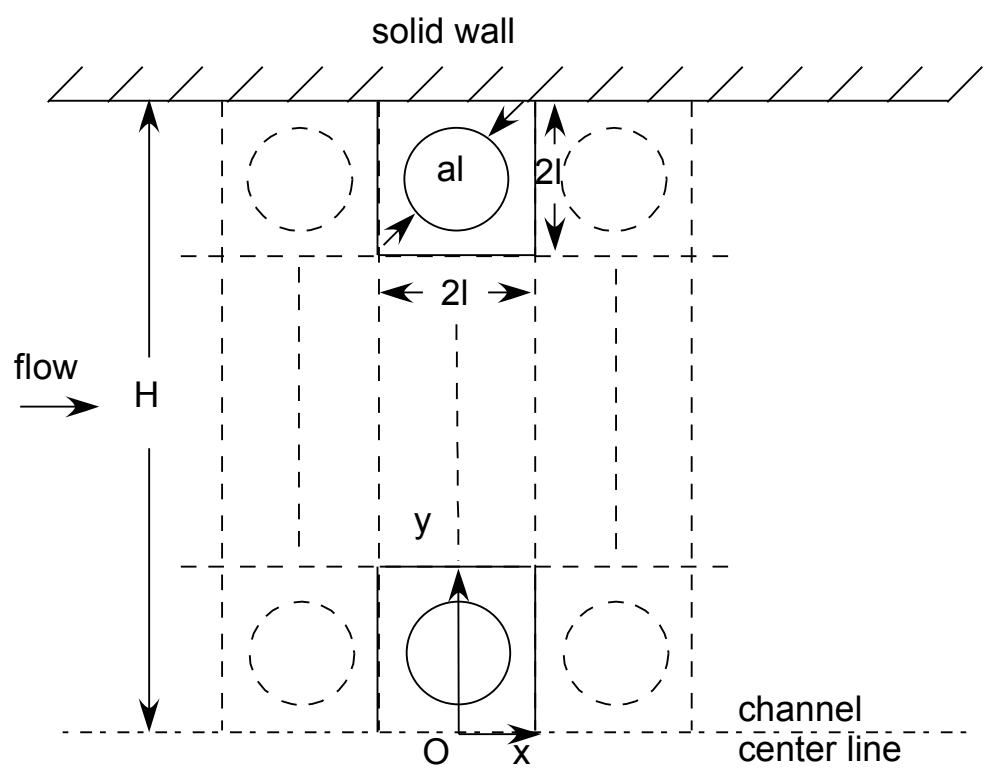



(b)

(a)

Figure 2. (a) Coordinates for formulation, Eq.(8).

(b) Typical computational grid for a unit cell.

Flow, conditions of which lie between the two limits of Eqs.(5,6), is investigated numerically. Referring to Figure 2, governing equations and boundary conditions are,

$$
\begin{array}{ll}
\nabla \cdot \vec{V}=0, & 0=-\nabla p+\mu \nabla^{2} \vec{V} \quad \text { with } \vec{V}=u \cdot \hat{i}+v \cdot \hat{j} . \\
u=v=0 & \text { at }(x, y)=(-l \leq x \leq l, y=H) \text { and on cylinder surface, } \\
\frac{\partial u}{\partial x}=0 & \text { at }(x, y)=( \pm l, 0 \leq y \leq H), \\
\frac{\partial u}{\partial y}=v=0 & \text { at }(x, y)=(-l \leq x \leq l, y=0) .
\end{array}
$$

Two commercially-available computational programs for hydrodynamic analyses are used in our numerical experiments, FLUENT (by Fluent Inc.) and FEMLAB (by COMSOL Group). The former is used as the main program; while, the latter is employed to confirm results from the former. Eq.(8) is solved computationally as the porosity, $\phi$, and the length ratio, $H / l$, are varied systematically. The porosity represents a fraction of the flow field in the cross sectional area of a $(2 l \times 2 l)$ unit cell; while, the ratio, $H / l$ (integer), indicates a number of cell layers over the channel depth. Figure 3 is presented to depict the range of the porosity of our investigation for the case of $H / l=10$. For the high porosity channel flow of $\phi=99.99 \%$, the parabolic velocity profile of the viscous flow limit over the channel 
depth is disturbed by regularly placed arrays of small cylinders; while, at low porosity the flow may be approximated by the lubrication type flow through a narrow slot with the uniform Darcian flow velocity being modified by local geometric variations. Computations are performed in dimensional form under the following conditions:

$$
\mu=0.001[\mathrm{~kg} / \mathrm{m} \cdot \mathrm{s}], \quad l=0.05[\mathrm{~m}],
$$

$T($ temperature $)=288.16[K], \quad u_{m}=1 \times 10^{-4}[\mathrm{~m} / \mathrm{s}]$.

\begin{tabular}{|llllllllll}
\hline 0 & 0 & 0 & 0 & 0 & 0 & 0 & 0 & 0 & 0 \\
0 & 0 & 0 & 0 & 0 & 0 & 0 & 0 & 0 & 0 \\
0 & 0 & 0 & 0 & 0 & 0 & 0 & 0 & 0 & 0 \\
& & & & & & & & &
\end{tabular}

$\phi=99.2 \%$



$\phi=87.4 \%$

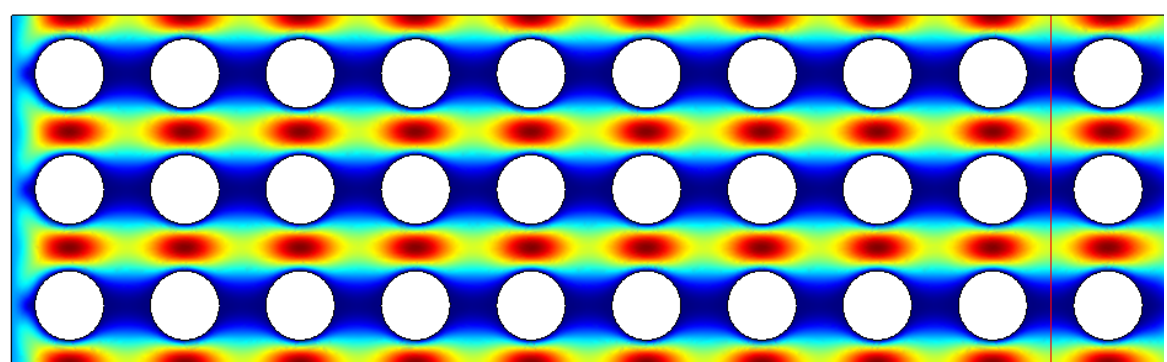

$\phi=71.7 \%$

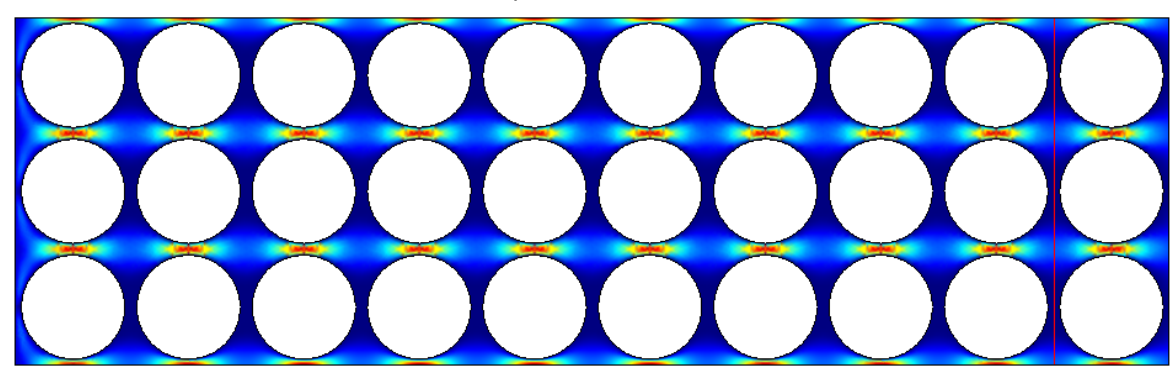

$\phi=36.4 \%$

Figure 3. Examples of flow field over a channel half-depth. 
The computational procedure is outlined below:

1. Solve Eq.(8) and find the $\mathrm{x}$-direction pressure gradient, $-d p / d x$ ), for a specified value of the porosity at the length ratio, $H / l=1$ (that is, the case of a single unit cell over the channel). (Actual computations are performed over multiple longitudinal cell columns (5 - 10) to ensure that the periodic conditions at the cross-sectional cell boundaries are satisfied accurately.)

2. Increase the value of $H / l$ by one, and repeat the computation.

3. In the case of $H / l=1$, the solid wall affects the entire flow field. As the magnitude of $H / l$ increases, cells near the center of the channel $(y=0$ in Figure 1) become less sensitive to the presence of the solid walls. Computation for a fixed value of porosity is terminated when the size of the wall-affected region becomes independent of $H / l$.

Sangani and Acrivos [15] reports solutions of the velocity field in a single unit cell (i.e. flow through square arrays of cylinders without solid bounding plates) by applying the least square collocation method [4] to a series solution, which satisfies a part of the required boundary conditions exactly. We used this solution for confirmation of the validity of our computational results as well as for determination of the required numerical conditions (number and size of the computational mesh as well as the conversion criteria). The validity of the computational results is confirmed by recovering the permeability, $K$, (Eq.(7)) reported in [15] over the range of 0.215 ( $\phi_{\text {minimum }}$ (cylinders in contact with each other) $<\phi<1$. When a unit cell becomes completely unaffected by the presence of the bounding walls, the velocity field should be identical to that of the solution in [15] everywhere in the cell. However, it is not easy to find the identity for the two-dimensional velocity field we are analyzing. Instead, the identity of the rate of entropy generation over a unit cell is used to find a degree of the wall effects on the flow field in the respective unit cell. The local volumetric rate of entropy generation over a unit width of the channel, $\dot{S}_{G}^{\prime / \prime}\left[\mathrm{W} / \mathrm{m}^{2} \cdot K\right]$, is related to the dissipation function $\Phi\left[1 / s^{2}\right][1]$. The rate of entropy generation per unit cell per width, $\dot{S}_{G, \text { cell }}^{\prime}$, is evaluated for each cell located over the channel half-depth by numerically integrating the following equation for volumetric rate of entropy generation due to the viscous dissipation,

$$
S_{G}^{\prime \prime \prime}=\frac{\mu}{T} \cdot \Phi \quad \text { where } \Phi=2\left(\left(\frac{\partial u}{\partial x}\right)^{2}+\left(\frac{\partial v}{\partial y}\right)^{2}\right)+\left(\frac{\partial u}{\partial y}+\frac{\partial v}{\partial x}\right)^{2}
$$

\section{Results and Discussion}

Figure 4 shows the axial velocity $(u)$ profile over the channel half-depth at a cross sectional boundary between two lateral cell columns (with the number of cylinders per lateral column $=$ four), as the porosity is varied. The centers of circular cylinders are located at $\mathrm{x}=l, 3 l, 5 l$ and $7 l$. The parabolic profile is computationally recovered for $\phi=100 \%$ (Figure 4(a)) as viscous flow between two parallel plates. Even at a very low solid fraction $\left(\phi=1-10^{-6} \%\right)$ (Figure $4(\mathrm{~b})$ ), the presence of cylinders is seen to affect the velocity profile substantially near the middle region of the channel; while, the velocity profile retains parabolic characteristics near the plate. It should also be noted that 
the effects of the cylinder adjacent to the wall (with its center at $x=l$ ) are more significant for the low porosity cases.
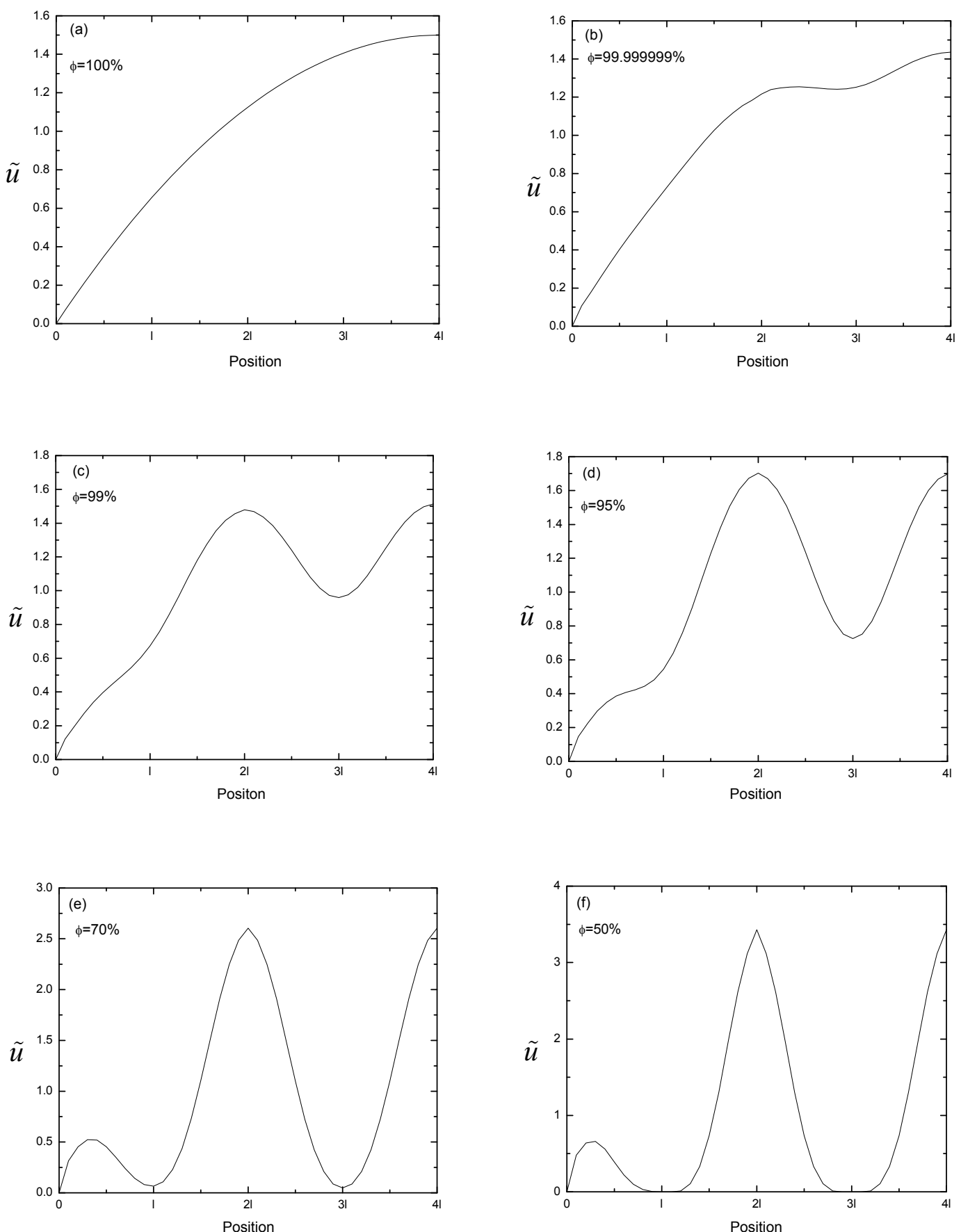

Figure 4. ( $\left.\tilde{u}=u \times 10^{4}[\mathrm{~m} / \mathrm{s}]\right)$ Axial velocity profile at the boundary normal to flow and between two neighboring cells: Effects of porosity. Number of cells $=4$, Volume flow rate $=4 \times 10^{3}\left[\mathrm{~m}^{3} / \mathrm{s}\right]$, Wall at $\mathrm{x}=0$. Profiles over channel half-depth. 

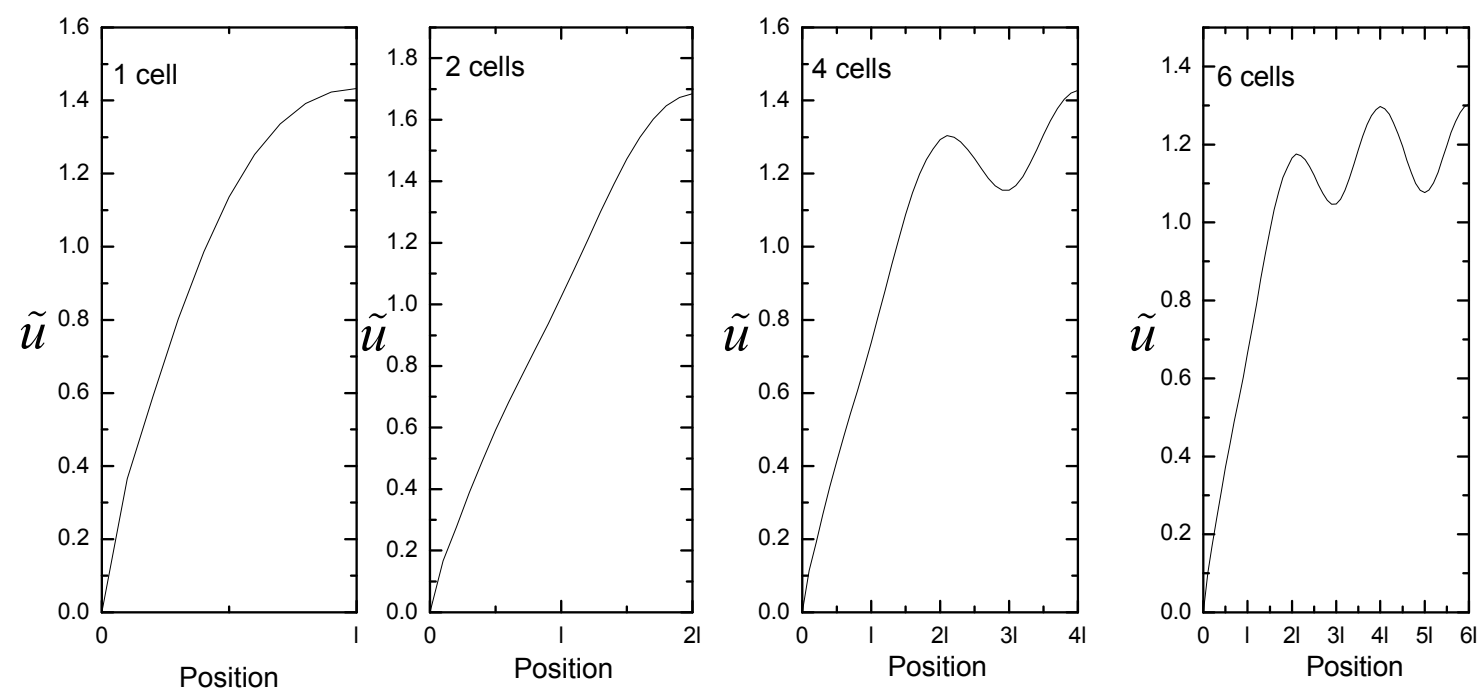

(a) $\Phi=99.99 \%$
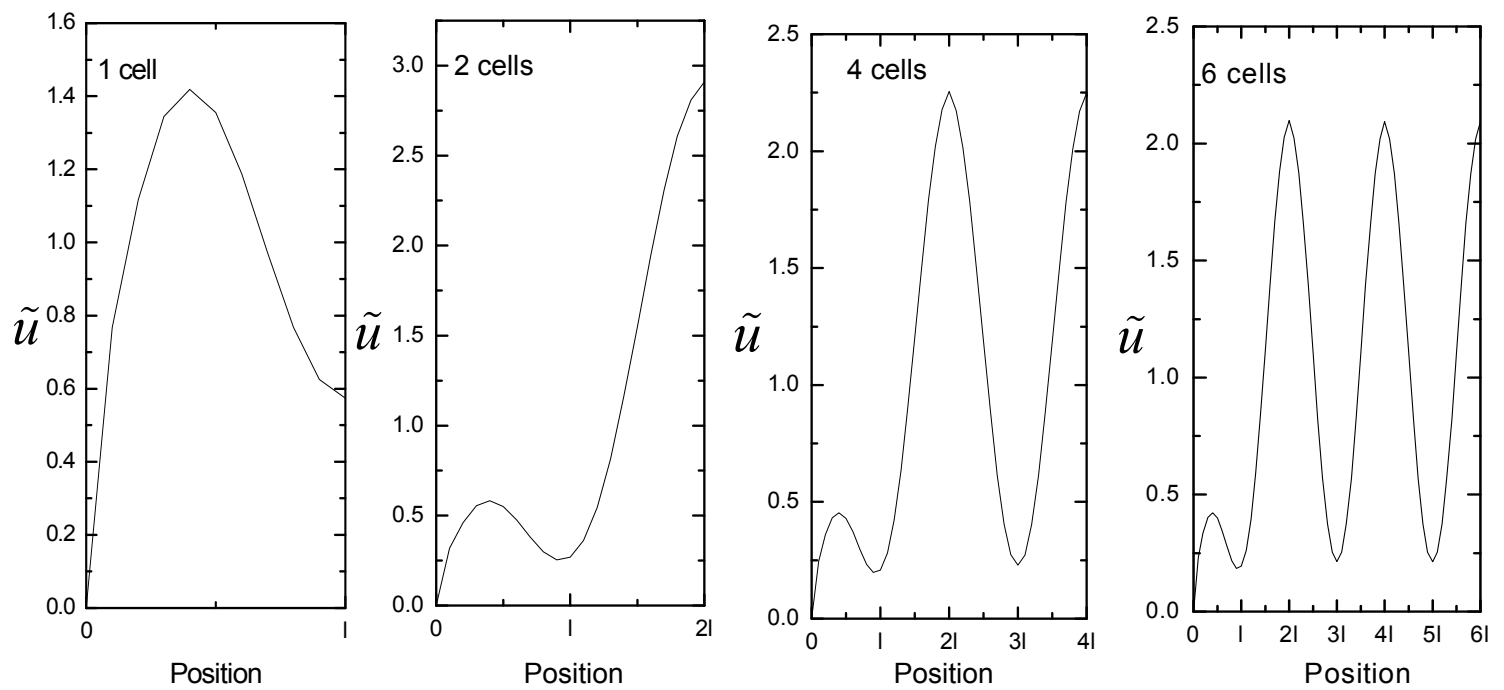

(b) $\Phi=80 \%$

Figure 5. ( $\left.\tilde{u}=u \times 10^{4}[\mathrm{~m} / \mathrm{s}]\right)$ Axial velocity profile at the boundary normal to flow and between two neighboring cells: Effects of number of cells. (a) $\phi=99.99 \%$, (b) $\phi=80 \%$. Volume flow rate $=$ Number of cells $\times 10^{-5}\left[\mathrm{~m}^{3} / \mathrm{s}\right]$, Wall at $\mathrm{x}=0$. Profiles over channel half-depth

In Figure 5, similar to Figure 4, the velocity profiles over the half-depth are presented to show the effects of the number of cells across the channel. Two cases are shown for $\phi=99.99 \%$ (Figure 5(a)), and $\phi=80.00 \%$ (Figure 5(b)). Large velocity changes around the cylinders compared to the corresponding changes near the plate (located at $x=0$ ) result in a smaller rate of entropy generation in the cell adjacent to the plate.

Table 1 lists computational results of the rate of entropy generation of each cell for the case of ten cells across the entire channel depth. Even at a very high porosity of $\phi=99.99 \%$, the plate effects are 
limited to the region within three cells from the plate wall with the affected region becoming even smaller as the porosity is decreased.

Table 1. Variation of $\dot{S}_{G, \text { cell }}^{\prime}$ over Channel Half-Width.

(Cell I in contact with the bounding plate at $\mathrm{y}=\mathrm{H}$ in Fig 2(a))

\begin{tabular}{|c|c|c|c|c|}
\hline & \multicolumn{2}{|c|}{$\dot{S}_{G, \text { Cell }}^{\prime}($ Cell i $) / \dot{S}_{G, \text { Cell }}^{\prime}($ Cell 5$)$} & \multicolumn{2}{|c|}{$(\mathrm{i}=1-5)$} \\
\hline & $\phi=85 \%$ & $95 \%$ & $99 \%$ & $99.99 \%$ \\
\hline Cell 1 & 0.639 & 0.647 & 0.656 & 0.692 \\
\hline Cell 2 & 0.999 & 0.995 & 0.981 & 0.927 \\
\hline Cell 3 & 1.0 & 0.999 & 0.999 & 0.997 \\
\hline Cell 4 & 1.0 & 1.0 & 1.0 & 1.0 \\
\hline Cell 5 & 1.0 & 1.0 & 1.0 & 1.0 \\
\hline
\end{tabular}

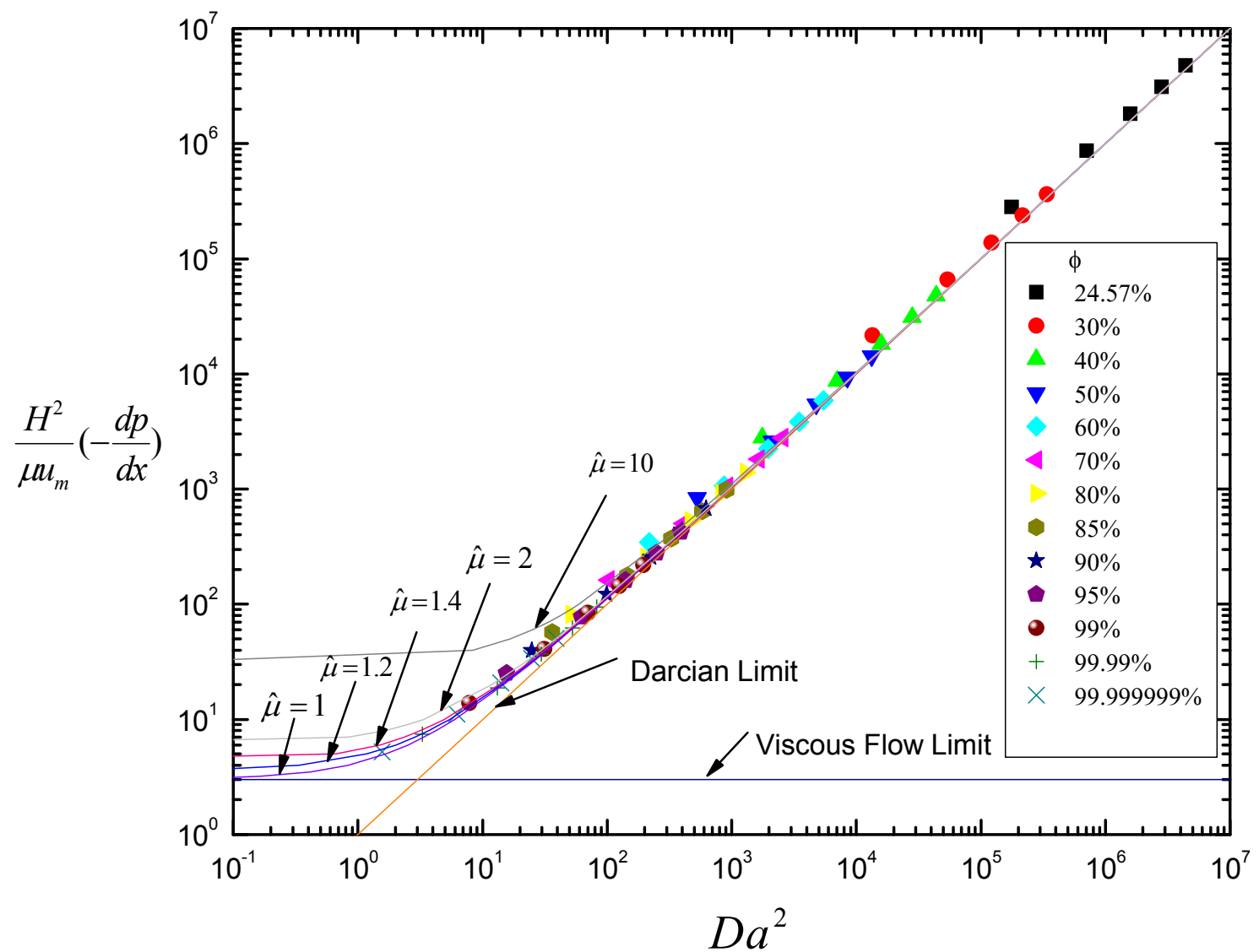

Figure 6. $\left(H^{2} / \mu u_{m}\right)(-d p / d x)$ vs $H^{2} / K$.

Figure 6 summarizes the computational results of the (non-dimensional) axial pressure gradient vs $D a^{2}$, Eq.(4), covering the porosity range up to $\phi=1-10^{-6} \%$. As was shown in Table 1 the bounding wall affects the flow up to $\sim 3$ unit cells away from the wall. The convergence of the non-dimensional pressure gradient to the Darcian limit is computationally confirmed, indicating that a critical value of 
$D a$ for convergence depends on the porosity. Computations are terminated for a specified value of $\phi$ upon reaching a condition sufficiently close to the Darcian limit. On the other hand, even at the solid fraction of $C=10^{-6} \%$, the viscous effect of the flow around the cylinder remains important with the minimum value of $D a^{2}$ being limited to $\sim 1.5$. (The computational lower limit is due to difficulties of the computational grid generation that is small in size and large in numbers sufficiently for accurate resolution of the velocity field near the cylinder.) The lowest value of $D a^{2}$ corresponds to the case of $H / l=1$ (a single cylinder over the channel width) for each set of numerical results with a fixed value of $\phi$. Also, results may not be presented in a continuous curve as an incremental change in the number of cylinders in the lateral direction leads to a step change in $D a^{2}$. Figure 6 indicates that, between the Darcian and the viscous flow limit, all numerical results may be recovered by setting $\hat{\mu}=1.0-1.2$ in Eq.(4). Our numerical range is limited to $H / l \leq 20$. Classical Darcy's law is valid under the assumption of very slow $(\mathrm{Re} \rightarrow 0)$ flow through a layer of a porous medium in which the local (microscopic) length scale ( $l$ in the present analysis) is sufficiently small compared to the overall (macroscopic) length scale ( $H$ ). For the two cases of (a) $\phi=50 \%$ and (b) $\phi=90 \%$, Figure 6 yields the following results:

(a) $\phi=50 \%, D a^{2}=\sim 5.0 \times 10^{6}$ for $H / l=20$;

$D a^{2}=\sim 1.0 \times 10^{4}$ as lower threshold for Darcian limit.

(b) $\phi=90 \%, D a^{2}=\sim 2.5 \times 10^{4}$ for $H / l=20$;

$D a^{2}=\sim 1.0 \times 10^{3}$ as lower threshold for Darcian limit.

A corresponding thermal problem had been analyzed $[2,6,16]$ for stability of a fluid saturated porous medium between two horizontal plates heated from below. Results, obtained under an assumption of $\mu_{e}=\mu(\hat{\mu}=1)$, indicate that the Darcian limit is reached in terms of the critical Rayleigh number for the onset of convection if $D a^{2} \geq 10^{3}$, and that the viscous flow limit is valid if $D a^{2} \leq 10^{-1}$.

Our computational results along with Figure 6 indicate that the Darcy- Brinkman equation with the viscosity ratio substantially different from unity fails to satisfy the non-slip condition at bounding walls, particularly under the high porosity condition of $D a^{2}<\sim 10^{3}$, and that the effect of the bounding walls on the flow structure, which is confined to a narrow region near the walls, may be approximated by a porous medium with $\hat{\mu}=1$. Figure 7 is a sketch of a three layer model, proposed to accommodate the wall effects in the analyses of a Brinkman porous medium with $\hat{\mu} \neq 1$. It consists of two layers near the bounding walls with $\hat{\mu}_{B}$ (viscosity ratio of the top and the bottom layer) $=1$, and a middle (interior) layer with $\hat{\mu}_{I}$ (viscosity ratio of the middle layer) $\neq 1$. A solution for a parallel flow through the three layers may be sought from the Darcy-Brinkman equation for the top $(H-L \leq y \leq H)$ as well as for the middle layer $(0 \leq y \leq H-L)$ under the conditions of the symmetry at $y=0$, the non-slip condition at $y=H$, and the velocity- and the shear stress-continuity at $y=H-L$. 
The result is,

$$
\begin{aligned}
& \frac{\left(H^{2} / \mu u_{m}\right) \cdot(-d p / d x)}{D a^{2}}=1-\frac{1}{D a} \cdot \frac{\sqrt{\hat{\mu}_{I}} \cdot X+Y}{1+\sqrt{\hat{\mu}_{I}} \cdot X \cdot Y} \\
& \text { where } X=\tanh \left(\frac{D a}{\sqrt{\hat{\mu}_{I}}}\left(1-\frac{L}{H}\right)\right), \quad y=\tanh \left(D a \cdot \frac{L}{H}\right)
\end{aligned}
$$

By setting $L / H=0$ and 1 in Eq.(10), Eq.(4) is recovered for a porous layer with $\hat{\mu}=\mu_{I}$ and with $\hat{\mu}=1$, respectively. The left hand side of Eq.(10) is the ratio of the non-dimensional pressure gradient of the three layer model to that of a single layer (= the Darcian limit of $L / H=0$ ). For a common pressure gradient, therefore, the mean velocity, $\mu_{m}$, of the three layer model is higher than that of the single layer. Greater the effective viscosity of the middle layer is relative to the fluid viscosity, more flow is channeled through the top (bottom) layer. The length scale of the top layer depth, $L$, is of the order of $6 l$ in Figure 2, implying that the depth ratio, $L / H$, depends on the characteristic length scale of the porous structure (such as $l$ in Figure 2) as well as on the macroscopic scale of the channel depth, $H$. Figure 8 shows Eq.(10) as $D a$ is varied with $L / H$ as third parameter for the case of $\hat{\mu}_{I}=10$. For a case in which the wall layer thickness, $L$, is $\sim 1 / 1000$ of the channel half-depth, $H$, an increase of $\sim 10 \%$ in the mean velocity across the channel is predicted at $D a^{2}=10^{3}$ due to a higher flow velocity in the wall layer. Although a substantial reduction of the wall effect is observed as the magnitude of $L / H$ is reduced from $10^{-3}$ to $10^{-4}$, the wall effects remain important even at $L / H=10^{-4}$ for $D a^{2}<\sim 10^{4}$.



Figure 7. Schematic Diagram of Three Layer Model. 




Figure 8. $\left(H^{2} / \mu u_{m}\right)(-d p / d x) / D a^{2}$ vs $D a^{2}$ of the three layer model with $\hat{\mu}_{I}=10$.

\section{Summary}

The effects of the bounding solid walls are examined numerically for flow through regular, square arrays of circular cylinders between two parallel plates. The lateral velocity profiles are altered substantially from the parabolic profile of the viscous flow due to sharp velocity gradients around the cylinders even for the solid fraction as low as $10^{-6} \%$. Between the two limits of the Darcian porous medium and the viscous flow, our computational results agree well with the Darcy-Brinkman equation with $\hat{\mu}=\sim 1$ in terms of the axial pressure gradient, satisfying the non-slip boundary conditions at the bounding walls. This implies that, for a porous medium for which the Brinkman term is needed to account for the local geometric heterogeneity (which is absent for regular arrays of solid objects), a degree of satisfying the boundary conditions is compromised, particularly for the cases in which $\hat{\mu}$ is substantially greater than unity. It is proposed that the non-slip condition at the bounding solid walls be accounted for by introducing a thin porous layer with $\hat{\mu}=1$ near the solid walls.

Finally, the study indicates that the local value of the entropy generation rate is effective in distinguishing the region near and away from the solid boundary in the hydrodynamic patterns.

\section{References}

1. Bejan, A., Entropy Generation Through Heat and Fluid Flow. John Wiley and Sons, 1982.

2. Cheng, P. Heat Transfer in Geothermal Systems. Adv. Heat Trans. 1987, 14, 1-105.

3. Durlofsky, L.; Brady, J.F. Analysis of the Brinkman equation as a model for flow in porous media. Phys. Fluids. 1987, 30, 3329-3340. 
4. Forsythe, G.E.; Malcom, A.; Moler, C.B. Computer Methods for Mathematical Computations. Prentice-Hall, New Jersey, 1977.

5. Givler, R.C.; Altobelli, S.A. A determination of the effective viscosity for the BrinkmanForchheimer flow model. J. Fluid Mech. 1994, 258, 355-370.

6. Katto, Y.; Masuoka, T. Criterion for the onset of convective flow in a fluid in a porous medium. Int. J. Heat Mass Transfer 1967, 10, 297-309.

7. Khaled, A.R.A.; Vafai, K. The role of porous media in modeling flow and heat transfer in biological tissues. Int. J. Heat Mass Transfer 2003, 46, 4989-5003.

8. Koplik, J.; Levine, H.; Zee, A. Viscosity renormalization in the Brinkman equation. Phys. Fluids 1983, 26, 2864-2870.

9. Liu, S.; Masliyah, J.H. Non-linear flows in porous media. J. Non-Newtonian Fluid Mech. 1999, 86, 229-252.

10. Lundgren, T.S. Slow flow through stationary random beds and suspensions of spheres. J. Fluid Mech. 1972, 51, 273-299.

11. Nield, D.A., The boundary correction for the Rayleigh-Darcy problem: limitations of the Brinkman equation. J. Fluid Mech., 128, 37-46, 1983.

12. Nield, D.A.; Bejan, A. Convection in Porous Media. Springer-Verlag New York, 1992.

13. Pries, A.R.; Secomb, T.W.; Jacobs, H.; Sperandio, M.; Osterloh, K.; Gaehtgens, P. Microvascular blood flow resistance: role of endothelial surface layer. Am. J. Physiol. 1997, 273, H2272-H2279.

14. Pries, A.R.; Secomb, T.W.; Gaehtgens, P. The endothelial surface layer. Pflugers Arch.- Eur. J. Physiol. 2000, 440, 653-666.

15. Sangani, A.S.; Acrivos, A. Slow flow past periodic arrays of cylinders with application to heat transfer. Int. J. Multiphase Flow 1982, 8, 193-206.

16. Walker, K.; Homsy, G.M. A note on convective instability in Boussinesq fluids and porous media. J. Heat Transfer 1977, 99, 338-339.

17. Weinbaum, S.; Zhang, X.; Han, Y.; Vink, H.; Cowin, S.C. Mechanotransduction and flow across the endothelial glycocalyx. PNAS 2003, 100, 7988-7995.

(C) 2007 by MDPI (http://www.mdpi.org). Reproduction is permitted for noncommercial purposes. 